\title{
Social determinants of adherence to COVID-19 preventive guidelines: a comprehensive review
}

\section{Zahra Jorjoran Shushtari ${ }^{1}{ }^{\oplus}$, Yahya Salimi $^{2}{ }^{\oplus}$, Sina Ahmadi $^{3}{ }^{\oplus}$, Nader Rajabi-Gilan $^{2}{ }^{\oplus}$, Marzieh Shirazikhah ${ }^{1}{ }^{\oplus}$, Akbar Biglarian $^{1}{ }^{\oplus}$, Ali Almasi ${ }^{2}{ }^{\oplus}$, Mohammad Ali Mohammadi Gharehghani ${ }^{4}$ (i)}

\footnotetext{
${ }^{1}$ Social Determinants of Health Research Center, University of Social Welfare and Rehabilitation Sciences, Tehran, Iran

${ }^{2}$ Social Development \& Health Promotion Research Center, Health Institute, Kermanshah University of Medical Sciences, Kermanshah, Iran

${ }^{3}$ Department of Social Welfare Management, University of Social Welfare and Rehabilitation Sciences, Tehran, Iran

${ }^{4}$ Social Welfare Management Research Center, University of Social Welfare and Rehabilitation Sciences, Tehran, Iran
}

Received: July 8, 2021

Revised: October 13, 2021

Accepted: November 16, 2021

Corresponding author:

Yahya Salimi

Social Development \& Health

Promotion Research Center,

Health Institute, Kermanshah

University of Medical Sciences,

PO Box: 6719851351,

Kermanshah, Iran

E-mail: yahya.salimi@kums.ac.ir

Co-Corresponding author:

Sina Ahmadi

Department of Social Welfare

Management, University

of Social Welfare and

Rehabilitation Sciences, PO Box

1985713834, Tehran, Iran

E-mail:sinaahmadi25@gmail.com

\section{ABSTRACT}

Adherence to coronavirus disease 2019 (COVID-19) preventive guidelines (ACPG) is an important strategy to control the COVID-19 pandemic effectively. The present study aimed to identify and summarize the social determinants of ACPG among the general population. A comprehensive review was performed from December 2019 to February 2021 through searching electronic databases. Two independent reviewers assessed and selected relevant studies. Next, the characteristics and main findings of the included studies were summarized. Finally, the World Health Organization's conceptual framework of social determinants of health was used to synthesize the identified social determinants of ACPG. Forty-one of 453 retrieved articles met the inclusion criteria. The study results showed different patterns of ACPG among various communities. Furthermore, 84 social determinants were identified and categorized into structural and intermediary determinants. ACPG is a set of complex behaviors associated with different individual sociodemographic and behavioral characteristics; living and working conditions; COVID-19 knowledge, attitudes, and risk perceptions; exposure to sources and information level; leisure activities; social support; trust; social norms; psychosocial wellbeing; socio-economic position; and the socio-economic and political context. Interventions to promote ACPG among the general population should consider the identified social determinants of ACPG.

Keywords: COVID-19; Guideline adherence; Preventive behavior; Preventive guidelines; Social determinants of health 


\section{Introduction}

The coronavirus disease 2019 (COVID-19) pandemic is the third deadly coronavirus outbreak that has occurred in less than 2 decades, after severe acute respiratory syndrome and Middle East respiratory syndrome. COVID-19 is one of the most important global public health concerns and priorities [1]. Despite the implementation of medical care approaches and technical solutions in the health sector, these solutions are not enough. In this respect, adherence to COVID-19 preventive guidelines (ACPG) such as wearing a face mask, washing one's hands regularly, following stayat-home orders, and maintaining physical distance are among the necessary important, simple, and cost-effective strategies for controlling the pandemic [2-4]. This strategy is particularly critical in low- and middle-income countries with limited medical and hospital resources. However, various factors at different levels-including the structural and psychosocial environment, as well as individual factors-may contribute to complete and efficient adherence to preventive guidelines; the consequent uneven distribution of adherence exacerbates social inequalities in health, especially in vulnerable groups [5]. Evidence has shown that perceptions of the risk of COVID-19 and the importance of ACPG vary based on political inclinations. In this respect, people with different political inclinations (e.g., Democrat rather than Republican in the USA) may have different attitudes and beliefs regarding risk levels and preferences for risk mitigation $[6,7]$. Some studies also reported that in societies where conspiracy theories are predominant, people are less likely to follow health-protective behaviors. Furthermore, previous research has linked crowded and unsanitary living conditions in shelters and marginalized suburbs, unsafe housing, a perception of insecurity in one's neighborhood, not having green space around one's house, less feeling comfortable at home, and low quality of life to lockdown adherence during the COVID-19 epidemic [8-11]. Marginalized people who live in crowded and unsanitary suburbs cannot wash their hands regularly due to insufficient access to tap water and sanitation at home. Moreover, they cannot adhere to physical distancing due to high population density. Precarious employment (such as having temporary and informal jobs, and limited access to social protection programs) is also associated with inequality in the risk of COVID-19 disease [12]. In this regard, evidence from the USA has shown a higher proportion of infection and mortality among African-Americans and Latinos than among other racial and ethnic groups [13,14].

The social determinants of health (SDH) refer to the social and environmental circumstances people live and work in, which shape exposure and vulnerabilities [15]. The SDH framework also provides a broad and comprehensive way to systematically assess the general population's ACPG. Applying the World Health Organization (WHO)'s conceptual framework of SDH helps recognize entry points for health planning and policy actions for current and future pandemics [16]. According to this conceptual framework, the socio-economic and political contexts of society (including governmental, economic, social, and public policies, as well as culture, and social values) place people in various socio-economic positions based on their education, occupation, income, gender, and race or ethnicity in a society [15]. These structural determinants, socio-economic and political contexts, and socio-economic status shape exposure to intermediary social determinants such as living and working conditions, behaviors, and psychosocial factors [15,17].

Although previous research has investigated the role of social factors in ACPG $[4,18]$, no studies in the literature have yet systematically reviewed the structural and immediate circumstances that may affect adherence to preventive guidelines among the general population, especially with the SDH approach [19-21]. Therefore, the present review study aimed to identify and summarize the social determinants of ACPG among the general population.

\section{Materials and Methods}

The present study comprehensively reviewed original articles related to social determinants of ACPG among the general population based on the Preferred Reporting Items for Systematic Reviews and Meta-Analyses (PRISMA) statement [22]. International databases such as PubMed, Medline via PubMed, Web of Science, Scopus, and the Google Scholar search engine were searched for articles published from December 2019 to February 2021.

A sensitive search strategy was applied to retrieve relevant studies. The Medical Subject Headings controlled vocabulary system was used to define the keywords. The following search strategy was used for PubMed, and equivalent formulations were used for the other databases: (COVID-19 OR "Covid outbreak" OR "Corona Virus") AND ("social factors" OR "social determinants" OR inequality OR "socio-economic status") AND (compliance OR adherence OR adoption) AND ("COVID-19 protocol" OR "COVID-19 guideline" OR "COVID-19 recommendations" OR "social distancing" OR "wearing a mask" OR "preventive behaviors" OR "selfisolation"). Based on the population/problem, exposure/ independent variable(s)/intervention, comparison, outcome, time (РECOT) framework, the included studies 
were observational, interventional, and qualitative works conducted among the general population that assessed the association between at least 1 social factor and ACPG; the search was also limited to studies that were peer-reviewed and published in English (Table 1). Studies that did not provide information on the pre-specified PECOT criteria were excluded. For data management, all the retrieved studies were imported into EndNote ver. $\mathrm{X}$.

Studies were screened and reviewed in 3 steps: title, abstract, and full-text review. After excluding duplicates, the titles of the retrieved studies were appraised. Next, 2 reviewers independently reviewed abstracts of the selected articles based on the inclusion criteria. Any disagreements between the 2 reviewers were resolved by consultation with a third reviewer. Finally, the full-texts of the related articles were reviewed, and relevant data were extracted.
We summarized the study location, sample size, study type, and main findings. Furthermore, based on the WHO conceptual framework of SDH, we categorized the findings of the included studies into structural and intermediary social determinants of ACPG.

\section{Results}

\section{Characteristics of Included Studies}

This review study was conducted in accordance with the PRISMA guidelines [22]. Figure 1 shows details of the process from the initial search and screening to the final study inclusion. In the initial search, 453 studies were retrieved and, after excluding duplicates, 152 articles were evaluated. Finally, 41 relevant articles were included based on the inclusion criteria. Two of the included studies were

Table 1. The PECOT criteria for the research question to identify the social determinants of ACPG

\begin{tabular}{lccccc}
\hline PECOT & Population & $\begin{array}{c}\text { Exposure/ } \\
\text { independent variable }\end{array}$ & Comparison & Outcome & Time \\
\hline The element of the question & General population & Social factors & Any comparator & ACPG & December 2019 to February 2021 \\
\hline
\end{tabular}

ACPG, adherence to COVID-19 preventive guidelines.

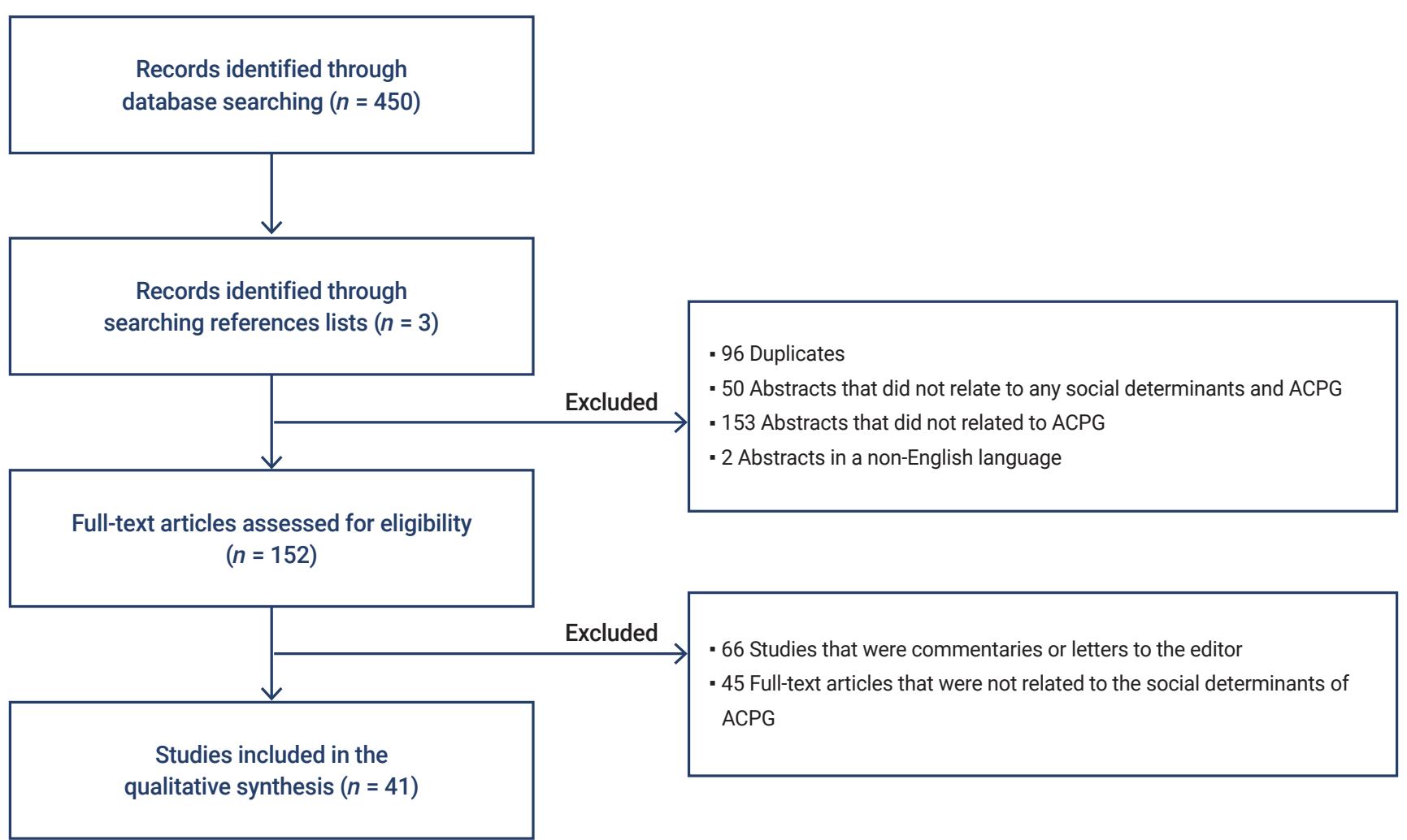

Figure 1. Preferred Reporting Items for Systematic Reviews and Meta-Analyses (PRISMA) flowchart of the search strategy. ACPG, adherence to coronavirus disease 2019 preventive guidelines. 
not peer-reviewed $[23,24]$. Among the retrieved studies, 66 were commentaries or letters to the editor, which were excluded although they addressed the role of social factors in general and SDH in particular (Figure 1).

Most of the included studies were cross-sectional, and 2 were longitudinal and prospective $[25,26]$. The included studies were conducted in various countries: 14 studies in the USA [6,24-37], 3 in Brazil [38-40], 8 in Asia [23,32,41-46], 5 in Africa [47-51], and the others in other countries. One study used international data [52], and 7 studies were conducted in more than 1 country [26,28,29,32,35,52,53]. Some studies mostly measured structural determinants of ACPG, while others focused only on intermediary determinants. Table 2 shows the summary characteristics of the all included studies [6,23-26,28-44,46-63]. Table S1 provides details of the selected studies [6,23-44,46-63].

After reviewing and analyzing the data, the social determinants of ACPG were extracted. Eventually, based on the WHO's SDH conceptual framework, they were categorized into structural and intermediary social determinants (Figure 2).

\section{Result of ACPG Status}

The status of ACPG varied in different communities. Various studies used different instruments to measure the status of ACPG, with most of them being self-developed measures. Many studies considered washing hands, wearing a face mask, not traveling to crowded places, adhering to quarantine, using disinfectants, not shaking hands, and social distancing as COVID-19 preventive guidelines [24,51,52,54,55]. A study in the USA also assessed social distancing by quantifying population movements within and across counties of origin by day [34].

A study in the USA showed that the average numbers of instances of hand-washing in the last 24 hours and leaving the house in the last 3 days were 13.2 times (standard deviation [SD], 13.5) and 2.5 times (SD, 4), respectively [24]. Another study in the USA found that most participants (84\%) reported wearing a mask in response to the COVID-19 pandemic [33]. In addition, a study in Indonesia reported relatively high adherence to some measures of COVID-19 preventive guidelines such as social distancing (87\%), using face masks (76\%), and hand-washing habits (87\%) [64]. In a study in Italy, Carlucci et al. [56] showed that respondents had medium to high adherence to preventive guidelines (mean, 32.59; SD, 5.22; range, 0 to 44), with an average quarantine duration of 15 days (SD, 6.64). The mean scores of adherence to preventive Polish governmental and WHO guidelines were 28.15 (SD, 7.11) and 23.99 (SD, 4.23), respectively, using 5-item and 4-item measures (7-point Likert scale) [57]. Azene et al. [51], in their study in Ethiopia, found that about half of the participants (48.96\%; 95\% confidence interval [CI], 45.05 to 52.89) had poor ACPG. The mean scores of adherence to preventive guidelines using a 10 -item 7-point Likert scale among Nigerians women and men were 25.52 (SD, 8.22) and 28.85 (SD, 10.53), respectively [49]. Moreover, a study in Uganda reported that the studied participants had high adherence to frequent hand-washing (96\%), physical distancing (90\%), and cough hygiene (86\%), whereas low adherence was reported for using masks (33\%) and disinfecting one's phone (42\%), laptop (26\%), bag (20\%), and TV remote control (18\%) [48].

\section{Social Determinants of Health and ACPG}

\section{Structural social determinants and ACPG Contextual factors}

The context of society, as the first element of structural social determinants, encompasses all economic, social, political, and cultural mechanisms that generate and maintain social hierarchies that influence health outcomes in a population [15]. According to our findings, political conservatism, political inclinations (e.g., Democrat, Republican, independent), political polarization, compact development (4 distinct dimensions of urban sprawl: development density, land use mix, population and employment centering, and street accessibility), geographical area (region/country and county), local economic endowments, communication of government/authorities (being clear and understandable, credible and honest, guided by the interests of the people regarding the COVID-19 crisis), governmental recommendations for home quarantine, religious beliefs, confidence in science, conspiracy beliefs, and fatalism were contextual factors associated with ACPG.

\section{Socio-economic position}

Socio-economic status is the second element of the structural social determinants defined by education, occupation, and income indicators. According to the WHO's conceptual framework, gender, race, or ethnicity are other social stratifiers that have intrinsic roles in determining people's position on a society's socio-economic ladder [15]. Some of the included studies showed that socio-economic profiles, including gender, education, household size, employment status, income, race, and ethnicity, were significantly associated with ACPG $[40,41]$. However, these associations were not reported in other studies $[48,54]$. In a study of social distance and inequality during the COVID-19 pandemic in the USA, Zhai et al. [27] found that although both rich and poor generally reduced their travel, poor people reduced their travel less than rich people, except for going to parks.

Some studies also reported that women exhibited more adherence to preventive guidelines than men $[24,42]$. Two 
Table 2. Summary of the studies included in the present review $(n=41)$

\begin{tabular}{|c|c|c|}
\hline Social determinants & Measured variables & Direction of association (positive/negative/null) \\
\hline \multicolumn{3}{|l|}{ Structural determinants } \\
\hline \multirow{12}{*}{$\begin{array}{l}\text { Socio-economic and political } \\
\text { context }\end{array}$} & Political conservatism & Negative [36] \\
\hline & Political inclinations & Positive [6] \\
\hline & Political polarization & Positive [6] \\
\hline & Compact development & Positive [25] \\
\hline & Geographical area & Positive [46] \\
\hline & Local economic endowments & Positive [34] \\
\hline & Communication of government/authorities & Positive [35] \\
\hline & $\begin{array}{l}\text { Governmental recommending for home } \\
\text { quarantine }\end{array}$ & Positive $[6,46]$ \\
\hline & Religiousbeliefs & Negative $[46,48,52]$ \\
\hline & Fatalism & Negative [36] \\
\hline & Conspiracy beliefs & Negative $[52,53,57,63]$ \\
\hline & Confidence in science & Positive [52] \\
\hline \multirow[t]{6}{*}{ Socio-economic position } & Education (higher education) & Positive $[6,25,38-41,46,47,53,55,56,60]$ \\
\hline & Occupation & Positive $[39,41,46,47,56,60]$ \\
\hline & Income & Positive $[23-25,34,36,38,40,41,46,48,53]$ \\
\hline & Gender (women) & Positive $[6,23,24,28,33,35,36,38-42,46-48,50,51,53-56,60,62]$ \\
\hline & Race & Positive $[6,24,33,36]$ \\
\hline & Ethnicity & Positive $[25,33,47]$ \\
\hline \multicolumn{3}{|l|}{ Intermediary determinants } \\
\hline \multirow[t]{2}{*}{ Living conditions } & Place of living & Positive $[24,31,35,39,46,48,56]$ \\
\hline & Housing quality & Positive [40] \\
\hline \multirow[t]{3}{*}{ Working conditions } & Type of work & Positive [38] \\
\hline & Work in non-governmental sectors & Negative [39] \\
\hline & Perceived ability to take sick leave & Positive [36] \\
\hline \multirow{5}{*}{$\begin{array}{l}\text { Individual demographic } \\
\text { characteristics and health } \\
\text { behaviors }\end{array}$} & Age (older people) & Positive $[6,23,24,30,35,36,38,39,41,46-48,50,53-56,60]$ \\
\hline & Marital status (being single/married) & (being single) Positive $[38,42]$ \\
\hline & & (being married) Positive $[35,43,46,48,56]$ \\
\hline & Smoking & Positive $[23,39]$ \\
\hline & Drug or alcohol abuse & Negative [29] \\
\hline \multirow{4}{*}{$\begin{array}{l}\text { COVID-19 knowledge, } \\
\text { attitudes, and risk } \\
\text { perceptions }\end{array}$} & Health literacy & Positive [53] \\
\hline & Knowledge & Positive $[30,32,36,42,49-51,59]$ \\
\hline & Attitudes & Positive $[30,32,36,42,49-51,59]$ \\
\hline & Risk perceptions & Positive $[30,42,50,51,59]$ \\
\hline \multirow{3}{*}{$\begin{array}{l}\text { Exposure to source and level } \\
\text { of COVID-19 information }\end{array}$} & Exposure to social media & Positive $[6,23,32]$ \\
\hline & Exposure to COVID-19 preventive information & Positive [23] \\
\hline & Source of COVID-19 information & Positive [48] \\
\hline $\begin{array}{l}\text { Attitude towards COVID-19 } \\
\text { preventive guidelines }\end{array}$ & $\begin{array}{l}\text { Attitude towards COVID-19 preventive } \\
\text { guidelines }\end{array}$ & Positive [23] \\
\hline \multirow{2}{*}{$\begin{array}{l}\text { Capacity and coping } \\
\text { appraisal }\end{array}$} & Capacity & Positive [30] \\
\hline & Coping appraisal & Positive [32] \\
\hline Leisure activities & Type of leisure activities (more active people) & Positive [43] \\
\hline Social norms & Social norms & Positive $[26,30,31,35,54,60]$ \\
\hline \multirow[t]{2}{*}{ Trust } & Trust in government & Positive [54] \\
\hline & Trust in social institutions & Positive [38] \\
\hline Psychosocial well-being & Psychosocial well-being & Positive $[23,53,61]$ \\
\hline Social support & Social support & Positive $[44,46,54,61,62]$ \\
\hline
\end{tabular}




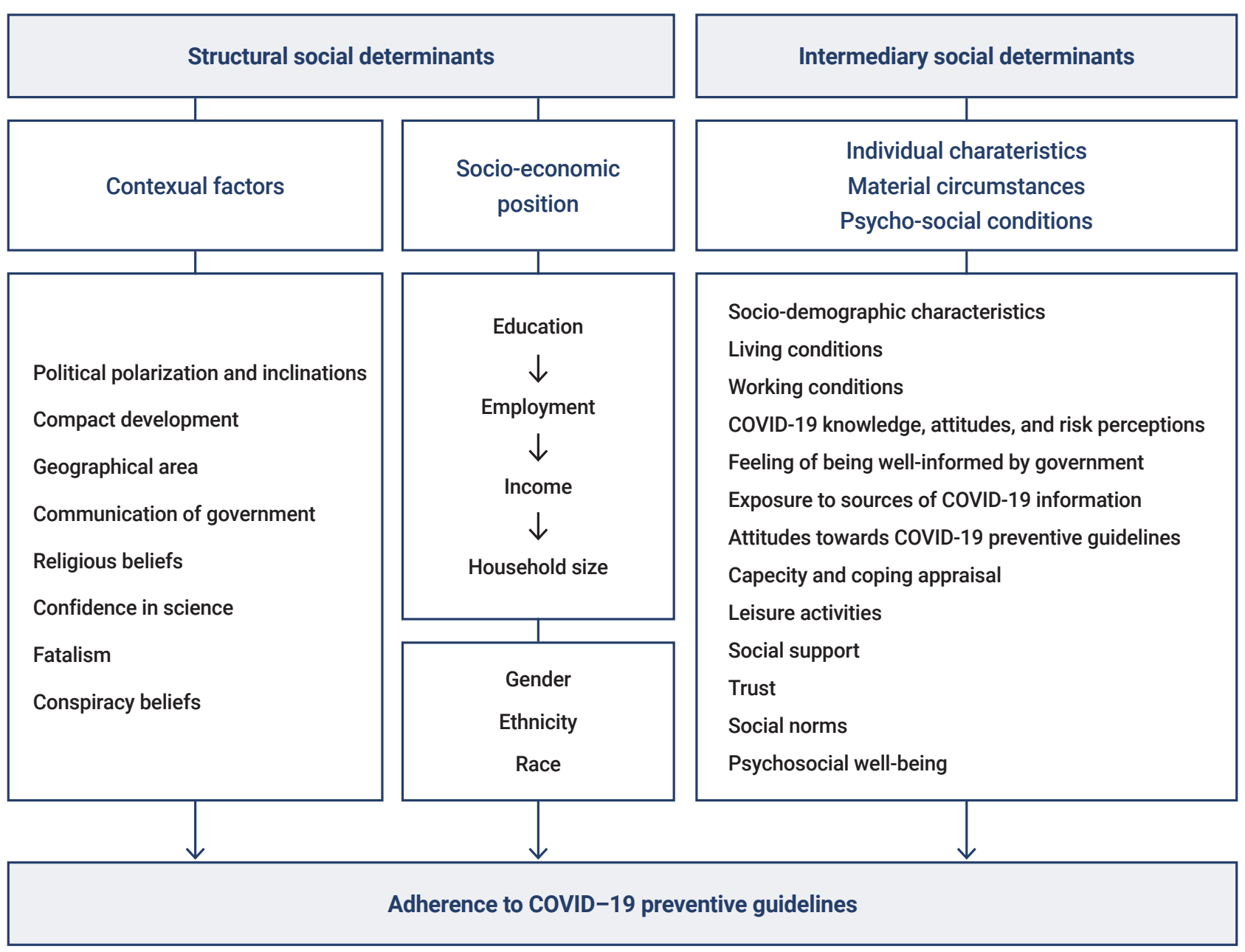

Figure 2. The social determinants of adherence to coronavirus disease 2019 (COVID-19) preventive guidelines in the present review.

The model was designed based on the World Health Organization's conceptual model of social determinants of health.

studies in Hong Kong showed that men were significantly less likely than women to engage in preventive behaviors, including washing their hands with soap and avoiding gatherings, not shaking hands, and avoiding contact with the elderly [41,55].

Furthermore, some studies showed that ethnicity and race were associated with ACPG [6,24,33]. However, 2 studies reported no association between ethnicity or race and ACPG [31,36]. A study in the USA found that race was one of the most important predictors of people's preventive behaviors, including hand-washing and traveling [24]. Accordingly, African-Americans and Hispanics were significantly more likely to wash their hands regularly than White people. However, compared to White people, AfricanAmericans were significantly more likely to stay at home [24].

\section{Intermediary Social Determinants and ACPG}

\section{Individual characteristics}

Demographic characteristics and health behaviors

In our review study, demographic characteristics, including age and marital status, were associated with ACPG $[24,41-43,54,55]$. There is conflicting evidence regarding the relationship between age and ACPG $[41,43,55]$. According to Kim and Cho [43], older people showed more preventive behaviors than young ones. On the contrary, a study in Hong Kong showed that young people had more preventive behaviors than older people, and the most preventive behavior was reported in the age group of 25 to 44 years [41]. Furthermore, the findings of the study by Cvetkovic et al. [55] in Serbia indicated that study participants in the age group of 28 to 39 years were more likely to limit their travel, store food for a month or more, and avoid contact with the 
elderly.

In contrast, people in the age group of 39 to 48 years were more likely to avoid hugging and kissing family members, friends, and acquaintances, use more disinfectants, and generally avoid contact with others. Study participants in the age group of 49 to 58 years also avoided handshaking, adhered to the recommended social distance, followed a proper diet, and avoided contact with pets [55]. A study in South Korea found that married people exhibited more ACPG than single people [43]. However, a study in Iran conversely reported that single people engaged in significantly more ACPG than married people [42].

Moreover, 3 studies showed that smoking and drug or alcohol abuse were associated with ACPG because smokers and users of alcohol or drugs were less likely to adhere to preventive guidelines. However, 1 study reported a nonsignificant association between smoking behavior and ACPG [46]. A study in Israel showed that people with a high level of past risk-taking behaviors were more likely to report nonadherence to the COVID-19 preventive guidelines (odds ratio [OR], $1.41 ; 95 \% \mathrm{CI}, 1.10$ to 1.81 ) [23].

COVID-19 knowledge, attitudes, and risk perceptions Individuals' knowledge, attitudes, and risk perceptions were associated with ACPG $[6,23,32,37,38,51,56,58]$. Some studies showed that health literacy [53] and knowledge about social distancing were associated with ACPG $[32,42,50,59]$. A study in Iran found that attitude toward COVID-19 risk was the strongest predictor of ACPG $(B=0.362, p=0.009)$ [42]. The results of an international study showed that COVID-19 risk perception had a significant direct association with ACPG $(B=0.35, p<0.001)$ [52]. However, a study in the USA reported no association between COVID-19 risk perception and ACPG [31]. Furthermore, a study in Ethiopia found a significant negative association in this regard (OR, 0.61; 95\% CI, 0.41 to 0.92) [51].

\section{Exposure to the source and level of COVID-19 information}

Three studies assessed the role of social media in ACPG. Two of them reported a significant association between exposure to social media and ACPG $[6,32]$, but the third study did not find a significant association [23]. A study in Ethiopia found that people who had more exposure to COVID-19 preventive information were more likely to adhere to COVID-19 preventive behaviors (OR, 1.85; 95\% CI, 1.03 to 2.43) [51]. A study also showed that the source of COVID-19 information was associated with ACPG [48]. More specifically, people who obtained COVID-19 information from healthcare workers (OR, 1.2; 95\% CI, 1.01 to 1.5 ) and village leaders (OR, 1.4; 95\% CI, 1.02 to 1.9) were more likely to adhere to COVID-19 preventive behaviors [48].

\section{Attitudes towards COVID-19 preventive guidelines}

Three studies found a significant association between attitudes towards COVID-19 preventive guidelines and adherence $[23,51,60]$. People who considered the preventive instructions effective and had positive attitudes about them reported more ACPG.

\section{Material circumstances}

\section{Living conditions}

The present review study indicated that place of living and housing quality were related to ACPG. A study in Brazil found that housing quality was related to keeping physical distance and adhering to stay-at-home orders during the COVID-19 quarantine period [40]. Some of the included studies that assessed variables related to respondents' place of living showed that people who lived in a large city and central states reported more ACPG [35,48,50,56].

\section{Working conditions}

Our study also revealed that working conditions were associated with ACPG $[12,36,39,65]$. Health workers were more likely to adhere to preventive guidelines [56], whereas those working in non-governmental sectors were less likely to adhere to preventive guidelines [39]. In a study in the USA, workers who had a greater perceived ability to take sick leave reported more adherence to hand-washing and physical distancing [36].

\section{Psychosocial conditions}

\section{Capacity and coping appraisal}

Regarding the capacity variable, only 1 study assessed the role of capacity on ACPG [30]. This study defined capacity as "I have the ability to do this" and found that capacity was the main predictor of maintaining social distancing $(B=0.09$; $95 \% \mathrm{CI}, 0.0$ to 0.27$)$ and intention to stay at home $(\mathrm{B}=0.63$; $95 \% \mathrm{CI}, 0.46$ to 0.80 ) [30]. One study also showed that coping appraisal was associated with social distancing [32].

\section{Leisure activities}

Only 1 of the included studies assessed the role of the type of leisure activities in ACPG [43]. That study showed that people who generally participated in cultural and art activities in their leisure time (mean, 4.275; SD, 0.499) and those involved in social (mean, 4.249; SD, 0.525) and tourism-related activities (mean, 4.223; SD, 0.482) exhibited 
more adherence to preventive guidelines [43]. Furthermore, those who participated in leisure activities along with their families showed high preventive behaviors. In contrast, those who spent their leisure time with friends of the opposite gender had low levels of precautionary behaviors [43].

\section{Social norms}

Only 5 studies showed significant positive associations between social norms and ACPG $[26,30,31,54,60]$. One of the studies, which used data from 2 cross-sectional surveys ( $n=2,000$ in survey 1; and 2,003 in survey 2$)$ in France, reported that social norms were significantly associated with ACPG $(B=0.13, p<0.001$ for survey $1 ; B=0.33, p<0.001$ for survey 2) [54].

\section{Trust}

This intermediary social determinant subcategory is related to 2 concepts: trust in overnment and trust in social institutions (e.g., health workers, media, and hospitals). A study that used data from 2 cross-sectional studies $(n=2,000$ in survey 1; and 2,003 in survey 2$)$ in France showed that trust in government $(\beta=0.07, p<0.01$ for survey $1 ; \beta=0.08, p<0.01$ for survey 2) was significantly associated with ACPG [54]. Another study in Brazil found that trust in government ( $B=-1.200 ; 95 \% C I,-1.600$ to -0.940$)$, health workers ( $\mathrm{B}=1.100 ; 95 \% \mathrm{CI},-0.770$ to 1.500 ), and media ( $B=0.550 ; 95 \% C I, 0.280$ to 0.820 ) were significantly associated with ACPG. Meanwhile, trust in hospitals did not show a significant association [38].

\section{Psychosocial well-being}

Only 1 study in Belgium showed that people who had low psychosocial well-being, including anxiety $(\mathrm{B}=1.85 ; 95 \% \mathrm{CI}$, 0.62 to 3.09 ), depression ( $B=2.99 ; 95 \% C I, 1.72$ to 4.26 ), anger $(B=2.74 ; 95 \% C I, 1.28$ to 4.21$)$, and social isolation $(B=2.82$; 95\% CI, 1.58 to 4.05 ) reported less ACPG [61].

\section{Social support}

Social support was another determinant that significantly affected people's ACPG $[44,61]$. Depending on the source (friends/ family), social support decreased or increased adherence to self-quarantine at home. Thus, perceived social support from one's family reduced the likelihood of poor adherence to stay-at-home orders (OR, 0.874; 95\% CI, 0.803 to 0.950 ). However, the association between perceived social support from friends and adherence to stay-at-home orders was not significant (OR, 0.926; 95\% CI, 0.849 to 1.010) [44].

\section{Discussion}

Although excellent progress has been made in vaccination, the best way to deal with this pandemic is adherence to preventive guidelines $[66,67]$. Successful implementation of the guidelines requires substantial engagement from citizens and communities.

\section{ACPG Status}

This review study aimed to identify the social determinants of ACPG in the general population. Our findings show that the status of ACPG varied in different communities. In some studies, adherence was high on average, while other studies reported moderate or low adherence. Interestingly, the findings of the review study indicated that higher-income countries such as the USA, France, and Canada had higher levels of adherence than low- and middle-income countries such as Uganda, Nigeria, and Indonesia. These differences may be related to various individual, communities, sociocultural, economic, and structural factors.

\section{Social Determinants of Health and ACPG}

\section{Structural social determinants \\ Contextual factors}

The results of our review showed that at the level of structural social determinants, political inclinations and polarization were associated with ACPG. One study in the USA found that Democrats were 1.76 times more likely than Republicans to wear a face mask and 1.45 times more likely to avoid public spaces or crowds [6]. Participants identified as independent were less likely to wear a face mask than Republicans, though they were 1.23 times more likely to avoid public spaces or crowds [6]. One explanation for this finding is that people who have different political inclinations within a politicized society may follow different sources of information and guidance about COVID-19. Hence, they react differently towards risks, governmental preventive guidelines, and the necessity of adhering to preventive behaviors. This finding may also reflect different approaches and reactions of various political groups about collective strategies (as opposed to individual efforts) in society to promote health outcomes.

Our results also indicate that compact development and geographical areas were associated with ACPG [25,62]. People who live in developed areas may have better access to online shopping options and health information, which minimizes their traveling needs and affects their protective behaviors, respectively.

Local economic endowments were another structural 
social determinant of ACPG in our study [34]. In societies with low levels of economic development, the health share of the gross domestic product and the level of government health expenditures are often low. Therefore, these countries seem to face many problems, such as providing and equally distributing hygiene materials (e.g., face masks, disinfectants, and detergents) and improving access to health care to prevent and control this pandemic.

Moreover, we found that governments' communication and transparency of information flow were associated with ACPG. One explanation for this finding is that a clear and strong relationship between the government and populace, regardless of economic development and gross domestic product, can increase trust in society and convince people to engage in more ACPG. Although government communication, as the main source of social influence, can significantly affect the adoption of preventive behaviors, it may negatively affect the further spread of disease in society. These findings are consistent with the results of other studies that addressed the role of socio-economic factors in preventive behaviors [68-70] and emphasized the necessity for comprehensive and health-oriented approaches by governments, especially in conditions of limited resources. Furthermore, our findings indicate that conspiracy beliefs are a substantial barrier to ACPG. In this respect, messages from authorities and local governments denouncing or enhancing conspiracy beliefs may be effective not only as a strategy for pandemic management by the state, but also for attitudes and behaviors at the individual level. For example, in communities with a conspiracy theory-based approach to the pandemic, the government may prioritize political decisions over health decisions. Therefore, instead of focusing on optimal pandemic management at home and emphasizing quarantine, the government may seek to maintain its political position and authority in the world and manage health within a political framework. Furthermore, in communities where conspiracy beliefs regarding the pandemic are predominant, people are less likely to engage in COVID-19 preventive behaviors. This finding aligns with previous studies that found associations between conspiracy beliefs and reluctance to follow health guidelines [71-73].

\section{Socio-economic position}

Some of the included studies showed significant associations between socio-economic position or its indicators and ACPG. Socio-economic position may affect ACPG in several ways and engender differential exposures to COVID-19. People with low education may not have sufficient knowledge about the disease and preventive health behaviors [74]. The knowledge and skills acquired through high education levels may improve people's cognitive assessment and perceptions of health messages about COVID-19, enabling them to use those messages in practice [15]. Furthermore, lower-income people usually work in temporary and unstable jobs that make it impossible to work remotely, even in the COVID-19 pandemic [75]. They may also experience some difficulties in obtaining the necessary preventive equipment such as face masks, gloves, disinfectants, and detergents, thereby not following the stay-at-home order and preventive guidelines properly. These people may live in small, unsanitary, and crowded homes, which reduces the possibility of having adequate personal space and maintaining social distance. This finding suggests that the government should support programs by providing packages for individuals of low socio-economic and educational status to help increase ACPG in this population.

Many studies emphasized gender differences in compliance with protective guidelines $[24,35,38,46,53,54,60]$, finding that men were less likely to follow these guidelines than women. This finding may be related to gender roles, norms, and social rules that determine men's and women's responsibilities, expectations, opportunities, limitations, and behaviors in society [76]. Due to masculinity stereotypes, men often pretend that they are immune against disease, deny illness or weakness, present themselves as invulnerable, engage in risky behaviors, and are unwilling to seek health care services [76]. Gender stereotypes and differential expectations about men's role in society, linked to their primary duties in providing their families' livelihood and the necessity of maintaining their jobs and earning income, lead to a greater presence in society than that of women. Thus, men may underestimate the risk of contracting COVID-19 and pay less attention to preventive guidelines such as staying at home. This finding highlights the necessity of genderbased educational programs about COVID-19 preventive guidelines as one of the most important policies, in which men's and women's roles and responsibilities are clarified separately.

Race or ethnicity, as another indicator of socio-economic position acting as a social stratifier [15], was associated with ACPG $[6,24,33]$. A study in the USA reported that AfricanAmericans and Hispanics were more likely to wash their hands regularly than White people; however, AfricanAmericans were less likely to stay home than individuals belonging to other races [24]. One explanation for this finding is that the value of health behaviors in different societies and the degree to which health is considered a collective social concern may differ among ethnic groups and communities. People's attitudes and behaviors are guided by the culture, implicit or explicit social values, 
and customs within a community, which may affect the adoption of COVID-19 preventive behaviors.

However, some of the included studies that assessed socio-economic position or its indicators did not find any significant associations with ACPG $[23,47,48,54,62]$. This inconsistency may have several reasons, such as heterogeneity in the studied populations, the use of various instruments for measurements, and measurement errors. In this sense, some studies measured socio-economic position at the county or country level and used aggregated measures, while some others measured this variable at the individual level.

\section{Intermediary Social Determinants}

\section{Individual characteristics}

Our review study showed that patterns of ACPG were different based on age and marital status. Although some studies showed that ACPG was higher among young people [41], other research found that ACPG increased with age [55]. This inconsistency might be due to social context and shared beliefs in different societies. One explanation is that high-risk perceptions and more perceived vulnerability among the elderly may lead to higher adherence. Conversely, the common belief that young people will not contract COVID-19 may lead to less ACPG. Regarding marital status, the included studies reported higher ACPG among married people than among single people. Since married people may consider themselves responsible for the health of their spouses and children, they are more likely to follow preventive guidelines than single people. Some studies also showed that smokers and users of alcohol or drugs were less likely to adhere to preventive guidelines. However, a study reported a non-significant association between smoking behavior and ACPG [46]. This finding suggests the necessity of paying particular attention to these at-risk people for COVID-19 prevention interventions.

We found an inconsistent association between COVID-19 risk perceptions and ACPG. Although some studies showed significant positive associations between COVID-19 risk perceptions and ACPG, a study in Ethiopia surprisingly reported that people who had higher levels of risk perception were less likely to adhere to preventive guidelines. Another study found no association in this regard. A plausible explanation for this inconsistency might be the use of various tools to measure the variables, differences in the study populations, and discrepancies in when the studies were conducted during the COVID-19 pandemic. Risk perception as a subjective judgment can help people find ways to cope with the COVID-19 pandemic. However, the dynamic properties of risk perception may vary during different pandemic stages, thereby affecting people's levels of protective behavior. This finding suggests that continuing interventions are necessary to enhance risk perceptions and correct misperceptions.

Our review study showed that providing timely and accurate information to society is necessary to address the COVID-19 pandemic successfully. The source of information was also associated with ACPG [48]. During the pandemic, various sources of information and powerful tools such as online social networks may create patterns of misinformation that have been dubbed an "infodemic" [68,77]. Misinformation and disinformation can result in low ACPG and reduce effective public health responses to the epidemic. A study in Uganda found that village leaders had an important role in providing COVID-19 information and that people who received information about COVID-19 preventive behaviors from village leaders were more likely to adhere to those behaviors [48]. This finding highlights the role of opinion leaders for the future effective implementation of health information interventions in communities.

\section{Material circumstances}

Living conditions, quality of housing, and place of living were associated with ACPG in our review study. Due to the nature of COVID-19 and its rapid transmission, limited access to some facilities (such as tap water, hot water, bathrooms, and toilets inside the house) creates some obstacles for ACPG, especially among people who are in lower socio-economic positions. According to the literature, people who live in shelters or crowded suburbs cannot wash their hands regularly due to insufficient access to tap water and sanitation at home [8,9]. Furthermore, living in low-quality housing without adequate space and facilities may reduce adherence to stay-at-home orders. A study in Mexico reported that 3.4 million people in the Mexican population did not have sufficient and proper space in their house to maintain a physical distance, especially if there was a family member with suspected or confirmed COVID-19 at home. In addition, 6.9 million people did not have adequate access to necessary housing facilities such as running water and sanitation. Another finding is that 13.9\% had dirt floors, and 58.8\% cooked their food with wood or charcoal. Therefore, the possibility of preventive measures such as regular hand-washing was minimal [78].

Work conditions (i.e., the work type, work environment, and providing sick leave) were other factors associated with ACPG in our study. One explanation is that people who work in high-risk conditions (such as health workers) may follow 
preventive guidelines more closely regarding higher risk perception and communication. Moreover, a supportive work environment based on a mutual understanding between the employer and employees can enhance the motivation and commitment of people to engage more actively in preventive behaviors to protect not only their health but also that of others. People who have temporary, informal, and non-governmental jobs often do not have enough support from their employers to take sick leave, access to the necessary protective equipment (such as gloves, masks, or alcohol), or reduced working hours in the COVID-19 pandemic [79]. These conditions may affect their intention and ability to implement preventive guidelines. These employees are at risk of contracting COVID-19 at their workplace, but they may also transmit the virus to others in the community [77]. Our finding suggests the need to apply educational interventions to inform employers about the role of working conditions and the benefits of providing support for their employees in adhering to the COVID-19 guidelines and stopping the spread of the virus at their workplaces and in the community.

\section{Psychosocial conditions}

Two of the included studies found that capacity and coping appraisal were major predictors of adherence to social distancing and stay-at-home orders [30,32]. These findings suggest that interventional programs for capacity building and improving coping are necessary for effective adherence to preventive guidelines in the community.

As another intermediary social determinant, social norms were also positively associated with ACPG $[26,30,31,54,60]$. This finding indicates that people's perceptions of attitude and behaviors of others, especially those in their social networks, can affect their adoption of protective behaviors in a community. Planning interventions that target people's social networks and opinion leaders in communities can encourage people to engage in more ACPG.

Two studies assessed the role of trust in government and social institutions in ACPG $[38,54]$. Although a study in the USA reported that people who had more trust in government were more likely to report more protective behaviors [54], Storopoli et al. [38] in Brazil showed a negative association between trust in the government and ACPG. However, Storopoli et al. [38] also found a significant interaction between vulnerability and trust in the government in relation to ACPG. The magnitude of the association between trust in the government and ACPG can increase or decrease based on levels of perceived vulnerability. This finding highlights that divergences in governmental policies in an emergency like the COVID-19 pandemic can harm people's health. Policy- makers should actively provide valid information, build trust, and increase community engagement to enhance ACPG.

During the COVID-19 pandemic, people may experience various mental health problems such as stress, anxiety, and depression, affecting ACPG. Beeckman et al. [61] reported that psychosocial well-being was negatively associated with ACPG. Our finding underscores the necessity of delivering psychosocial interventions for the community, which may help people cope with emerging challenges and enhance their protective behaviors.

Social support was another intermediary social determinant of ACPG [44,61]. Support from family and friends can buffer people's psychological stress caused by the fear of becoming sick and losing their job and income. Family support also may provide a safe and pleasant environment at home, especially during the lockdown period, which can increase people's resiliency in terms of following stay-at-home orders. To improve ACPG, steps should be taken to strengthen encouraging and supportive relationships between people and their family members.

The WHO conceptual framework emphasizes the interconnectedness of SDH, which play a decisive role during health-compromising conditions such as the COVID-19 pandemic. Although our goal was not to focus on indirect associations between social determinants and ACPG, and the included studies did not consider such relationships, it is nonetheless possible that the identified ACPG-related factors (from both the structural and intermediary levels) may be interrelated. For example, contextual factors such as fatalism and conspiracy beliefs can differently affect people's ACPG based on their income, education, occupation, gender, race/ethnicity, and socioeconomic status in general. As people with low education levels may be more strongly affected by fatalism and conspiracy theories, they may have lower COVID-19 risk perceptions, which in turn affects their tendency to engage in ACPG. Furthermore, differences in socio-economic positions can result in different exposures and vulnerabilities to health-damaging conditions (e.g., COVID-19). In this vein, people's education level can affect their occupation, which in turn determines their income level and also socio-economic status. Socio-economic status can affect people's leisure activities, working conditions (especially the possibility of telework), and living place, which in turn each can play an important roles in people's strategies for coping with the COVID-19 pandemic and their adherence to protective guidelines. Hence, future studies are suggested to assess both direct and indirect associations between social determinants and ACPG. 
The limitations of the current review are as follows. First, non-English and unpublished studies were not included in this study. Second, although the applied search strategy was broad, some articles may have been overlooked. Third, measurements of ACPG and its social determinants were not consistent across the included studies. This heterogeneity precluded us from performing a metaanalysis.

\section{Conclusion}

According to our findings, ACPG is a complex set of behaviors that are affected by various factors on different levels, including sociodemographic and individual lifestyle factors, social and community networks, living and working conditions, and the socio-economic and political context. Both structural and intermediary social determinants play a vital role in whether people follow COVID-19 protective guidelines. Our findings indicated that most determinants of ACPG were factors outside the health system. Therefore, effective management of this pandemic requires efforts to strengthen intersectoral collaboration and interventions beyond the reach of the health sector, particularly SDH. Governments and authorities should also make social policies to create an environment where people have fewer barriers to ACPG.

\section{Supplementary Material}

Table S1. Characteristics of related preliminary studies reviewed in the present study in detail $(n=41)$. Supplementary data are available at https://doi.org/10.24171/j.phrp.2021.0180.

\section{Notes}

\section{Ethics Approval}

Not applicable.

\section{Conflicts of Interest}

The authors have no conflicts of interest to declare.

\section{Funding}

None.

\section{Availability of Data}

All data extracted and analyzed during this study are included in this published article. For other data, these may be available through the corresponding author upon reasonable request.

\section{Authors' Contributions}

Conceptualization: all authors; Data curation: ZJS, YS, SA, MS, NRG; Formal analysis: ZJS, YS, SA; Investigation: all authors; Methodology: all authors; Project administration: ZJS; Resources: all authors; Supervision: YS, SA; Validation: all authors; Visualization: all author; Writing-original draft: ZJS, YS, SA; Writing-review 8 editing: all authors.

\section{Additional Contributions}

The authors are grateful to their colleagues for their help in preparing this paper.

\section{References}

1. World Health Organization (WHO). Coronavirus disease 2019 (COVID-19): situation report, 86. Geneva: WHO; 2020.

2. Markel H, Stern A, Cetron M. Nonpharmaceutical interventions implemented by us cities during the 1918-1919 influenza pandemic. Int J Infect Dis 2008;12:e432.

3. Jefferson T, Foxlee R, Del Mar C, et al. Physical interventions to interrupt or reduce the spread of respiratory viruses: systematic review. BMJ 2008;336:77-80.

4. Lee A, Cho J. The impact of epidemics on labor market: identifying victims of the Middle East Respiratory Syndrome in the Korean labor market. Int J Equity Health 2016;15:196.

5. Rangel JC, Ranade S, Sutcliffe P, et al. COVID-19 policy measures: advocating for the inclusion of the social determinants of health in modelling and decision making. J Eval Clin Pract 2020;26:1078-80.

6. Bruine de Bruin W, Saw HW, Goldman DP. Political polarization in US residents' COVID-19 risk perceptions, policy preferences, and protective behaviors. J Risk Uncertain 2020;61:177-94.

7. Dryhurst S, Schneider CR, Kerr J, et al. Risk perceptions of COVID-19 around the world. J Risk Res 2020;23:994-1006.

8. Lima NN, de Souza RI, Feitosa PW, et al. People experiencing homelessness: their potential exposure to COVID-19. Psychiatry Res 2020;288:112945

9. Patel JA, Nielsen FB, Badiani AA, et al. Poverty, inequality and COVID-19: the forgotten vulnerable. Public Health 2020;183:110-1.

10. Bellato A. A commentary on psychological factors affecting prosocial behaviors: what can we do to increase compliance with the regulations of physical distancing during the COVID-19 pandemic? Soc Sci Humanit Open 2020 May 5 [Epub]. http://doi.org/10.2139/ ssrn.3584414.

11. Raisi-Estabragh Z, McCracken C, Bethell MS, et al. Greater risk of severe COVID-19 in Black, Asian and Minority Ethnic populations is not explained by cardiometabolic, socioeconomic or behavioural factors, or by 25(OH)-vitamin D status: study of 1326 cases from the UK Biobank. J Public Health (Oxf) 2020;42:451-60.

12. Renzaho AM. The need for the right socio-economic and cultural fit in the COVID-19 response in sub-Saharan Africa: examining demographic, economic political, health, and socio-cultural differentials in COVID-19 morbidity and mortality. Int J Environ Res Public Health 2020;17:3445.

13. Thakur N, Lovinsky-Desir S, Bime C, et al. The structural and social determinants of the racial/ethnic disparities in the U.S. COVID-19 pandemic: what's our role? Am J Respir Crit Care Med 2020;202:943-9.

14. Shah M, Sachdeva M, Dodiuk-Gad RP. COVID-19 and racial disparities. J Am Acad Dermatol 2020;83:e35. 
15. Solar O, Irwin A. A conceptual framework for action on the social determinants of health. Social determinants of health discussion paper 2 (policy and practice). Geneva: World Health Organization; 2010.

16. Missoni E, Armocida B, Formenti B. Face masks for all and all for face masks in the COVID-19 pandemic: community level production to face the global shortage and shorten the epidemic. Disaster Med Public Health Prep 2021;15:e29-33.

17. World Health Organization (WHO). Commission on social determinants of health. Geneva: WHO; 2008.

18. Driedger SM, Maier R, Furgal C, et al. Factors influencing H1N1 vaccine behavior among Manitoba Metis in Canada: a qualitative study. BMC Public Health 2015;15:128.

19. Ahmad T, Khan M, Haroon, et al. COVID-19: zoonotic aspects. Travel Med Infect Dis 2020;36:101607.

20. Mamoon D. Health and economic outcomes of COVID 19. J Econ Soc Thought 2020;7:41-54.

21. Takian A, Raoofi A, Kazempour-Ardebili S. COVID-19 battle during the toughest sanctions against Iran. Lancet 2020;395:1035-6.

22. Moher D, Liberati A, Tetzlaff J, et al. Preferred reporting items for systematic reviews and meta-analyses: the PRISMA statement. Int J Surg 2010;8:336-41.

23. Pollak Y, Dayan H, Shoham R, et al. Predictors of non-adherence to public health instructions during the COVID-19 pandemic. Psychiatry Clin Neurosci 2020;74:602-4.

24. Alsan M, Stantcheva S, Yang D, et al. Disparities in coronavirus 2019 reported incidence, knowledge, and behavior among US adults. JAMA Netw Open 2020;3:e2012403.

25. Hamidi S, Zandiatashbar A. Compact development and adherence to stay-at-home order during the COVID-19 pandemic: a longitudinal investigation in the United States. Landsc Urban Plan 2021;205:103952.

26. Hagger MS, Smith SR, Keech JJ, et al. Predicting social distancing intention and behavior during the COVID-19 pandemic: an integrated social cognition model. Ann Behav Med 2020;54:713-27.

27. Zhai W, Liu M, Peng ZR. Social distancing and inequality in the United States amid COVID-19 outbreak. Environ Plan A Econ Space 2021;53:3-5.

28. Galasso V, Pons V, Profeta P, et al. Gender differences in COVID-19 attitudes and behavior: panel evidence from eight countries. Proc Natl Acad Sci U S A 2020;117:27285-91.

29. Taylor S, Paluszek MM, Rachor GS, et al. Substance use and abuse, COVID-19-related distress, and disregard for social distancing: a network analysis. Addict Behav 2021;114:106754.

30. Smith PH, Branscum PW. Feasibility, utility, and limitations of a rapid community behavioral diagnosis for social distancing during the 2020 coronavirus pandemic. Am J Health Promot 2021;35:77-83.

31. Bogg T, Milad E. Demographic, personality, and social cognition correlates of coronavirus guideline adherence in a U.S. sample. Health Psychol 2020;39:1026-36.

32. Al-Hasan A, Yim D, Khuntia J. Citizens' adherence to COVID-19 mitigation recommendations by the government: a 3-country comparative evaluation using web-based cross-sectional survey data. J Med Internet Res 2020;22:e20634.

33. Hearne BN, Nino MD. Understanding how race, ethnicity, and gender shape mask-wearing adherence during the COVID-19 pandemic: evidence from the COVID impact survey. J Racial Ethn Health Disparities 2021;1-8.

34. Wright AL, Sonin K, Driscoll J, et al. Poverty and economic dislocation reduce compliance with COVID-19 shelter-in-place protocols. J Econ Behav Organ 2020;180:544-54.

35. Margraf J, Brailovskaia J, Schneider S. Behavioral measures to fight COVID-19: an 8-country study of perceived usefulness, adherence and their predictors. PLoS One 2020;15:e0243523.

36. Jimenez T, Restar A, Helm PJ, et al. Fatalism in the context of COVID-19: perceiving coronavirus as a death sentence predicts reluctance to perform recommended preventive behaviors. SSM Popul Health 2020;11:100615.

37. Wise T, Zbozinek TD, Michelini G, et al. Changes in risk perception and self-reported protective behaviour during the first week of the COVID-19 pandemic in the United States. R Soc Open Sci 2020;7:200742

38. Storopoli J, Braga da Silva Neto WL, Mesch GS. Confidence in social institutions, perceived vulnerability and the adoption of recommended protective behaviors in Brazil during the COVID-19 pandemic. Soc Sci Med 2020;265:113477.

39. Faria de Moura Villela E, Lopez RV, Sato AP, et al. COVID-19 outbreak in Brazil: adherence to national preventive measures and impact on people's lives, an online survey. BMC Public Health 2021;21:152.

40. Bezerra AC, Silva CE, Soares FR, et al. Factors associated with people's behavior in social isolation during the COVID-19 pandemic. Cien Saude Colet 2020;25(suppl 1):2411-21.

41. Chan EY, Huang Z, Lo ES, et al. Sociodemographic predictors of health risk perception, attitude and behavior practices associated with health-emergency disaster risk management for biological hazards: the case of COVID-19 pandemic in Hong Kong, SAR China. Int J Environ Res Public Health 2020;17:3869.

42. Nasirzadeh M, Aligol M. Assessment of knowledge, attitude, and factors associated with the preventive behaviors of COVID-19 in Qom, Iran, in 2020. Qom Univ Med Sci J 2020;14:50-7.

43. Kim YJ, Cho JH. Correlation between preventive health behaviors and psycho-social health based on the leisure activities of South Koreans in the COVID-19 crisis. Int J Environ Res Public Health 2020;17:4066.

44. Paykani T, Zimet GD, Esmaeili R, et al. Perceived social support and compliance with stay-at-home orders during the COVID-19 outbreak: evidence from Iran. BMC Public Health 2020;20:1650.

45. Tong KK, Chen JH, Yu EW, et al. Adherence to COVID-19 precautionary measures: applying the health belief model and generalised social beliefs to a probability community sample. Appl Psychol Health Well Being 2020;12:1205-23.

46. Al-Sabbagh MQ Al-Ani A, Mafrachi B, et al. Predictors of adherence 
with home quarantine during COVID-19 crisis: the case of health belief model. Psychol Health Med 2021;1-13.

47. Ilesanmi O, Afolabi A. Perception and practices during the COVID-19 pandemic in an urban community in Nigeria: a cross-sectional study. PeerJ 2020;8:e10038.

48. Amodan BO, Bulage L, Katana E, et al. Level and determinants of adherence to COVID-19 preventive measures in the first stage of the outbreak in Uganda. Int J Environ Res Public Health 2020;17:8810.

49. Iorfa SK, Ottu IF, Oguntayo R, et al. COVID-19 knowledge, risk perception, and precautionary behavior among Nigerians: a moderated mediation approach. Front Psychol 2020;11:566773.

50. Mohamed AA, Elhassan EA, Mohamed AO, et al. Knowledge, attitude and practice of the Sudanese people towards COVID-19: an online survey. BMC Public Health 2021;21:274.

51. Azene ZN, Merid MW, Muluneh AG, et al. Adherence towards COVID-19 mitigation measures and its associated factors among Gondar City residents: a community-based cross-sectional study in Northwest Ethiopia. PLoS One 2020;15:e0244265.

52. Plohl N, Musil B. Modeling compliance with COVID-19 prevention guidelines: the critical role of trust in science. Psychol Health Med 2021;26:1-12.

53. Coroiu A, Moran C, Campbell T, et al. Barriers and facilitators of adherence to social distancing recommendations during COVID-19 among a large international sample of adults. PLoS One 2020;15:e0239795.

54. Raude J, Lecrique JM, Lasbeur L, et al. Determinants of preventive behaviors in response to the COVID-19 pandemic in France: comparing the sociocultural, psychosocial and social cognitive explanations. Front Psychol 2020;11:3345.

55. Cvetkovic VM, Nikolic N, Radovanovic Nenadic U, et al. Preparedness and preventive behaviors for a pandemic disaster caused by COVID-19 in Serbia. Int J Environ Res Public Health 2020;17:4124.

56. Carlucci L, D’Ambrosio I, Balsamo M. Demographic and attitudinal factors of adherence to quarantine guidelines during COVID-19: the Italian model. Front Psychol 2020;11:559288.

57. Kowalski J, Marchlewska M, Molenda Z, et al. Adherence to safety and self-isolation guidelines, conspiracy and paranoia-like beliefs during COVID-19 pandemic in Poland: associations and moderators. Psychiatry Res 2020;294:113540.

58. Yang XY, Gong RN, Sassine S, et al. Risk perception of COVID-19 infection and adherence to preventive measures among adolescents and young adults. Children (Basel) 2020;7:311.

59. Sturman D, Auton JC, Thacker J. Knowledge of social distancing measures and adherence to restrictions during the COVID-19 pandemic. Health Promot J Austr 2021;32:344-51.

60. Gouin JP, MacNeil S, Switzer A, et al. Socio-demographic, social, cognitive, and emotional correlates of adherence to physical distancing during the COVID-19 pandemic: a cross-sectional study. Can J Public Health 2021;112:17-28.

61. Beeckman M, De Paepe A, Van Alboom M, et al. Adherence to the physical distancing measures during the COVID-19 pandemic: a
HAPA-based perspective. Appl Psychol Health Well Being 2020; 12:1224-43.

62. Moussaoui LS, Ofosu ND, Desrichard O. Social psychological correlates of protective behaviours in the COVID-19 outbreak: evidence and recommendations from a nationally representative sample. Appl Psychol Health Well Being 2020;12:1183-204.

63. Allington D, Dhavan N. The relationship between conspiracy beliefs and compliance with public health guidance with regard to COVID-19. London: Centre for Countering Digital Hate; 2020.

64. Kasjono HS, Amalia R, Nugroho NM, et al. Study on physical distancing compliance towards corona virus risk (COVID-19). Eur J Mol Clin Med 2020;7:2236-42.

65. Truong N, Asare AO. Assessing the effect of socio-economic features of low-income communities and COVID-19 related cases: an empirical study of New York City. Glob Public Health 2021;16:1-16.

66. Young BE, Ong SW, Kalimuddin S, et al. Epidemiologic features and clinical course of patients infected with SARS-CoV-2 in Singapore. JAMA 2020;323:1488-94.

67. The Lancet. COVID-19: learning from experience. Lancet 2020;395:1011. 68. Adedimeji A, Sinayobye JD, Asiimwe-Kateera B, et al. Social contexts as mediator of risk behaviors in Rwandan men who have sex with men (MSM): implications for HIV and STI transmission. PLoS One 2019;14:e0211099.

69. Astrom AN, Rise J. Socio-economic differences in patterns of health and oral health behaviour in 25 year old Norwegians. Clin Oral Investig 2001;5:122-8.

70. Turrell G, Kavanagh AM. Socio-economic pathways to diet: modelling the association between socio-economic position and food purchasing behaviour. Public Health Nutr 2006;9:375-83.

71. Dunn AG, Surian D, Leask J, et al. Mapping information exposure on social media to explain differences in HPV vaccine coverage in the United States. Vaccine 2017;35:3033-40.

72. Jolley D, Douglas KM. The effects of anti-vaccine conspiracy theories on vaccination intentions. PLoS One 2014;9:e89177.

73. Thorburn S, Bogart LM. Conspiracy beliefs about birth control: barriers to pregnancy prevention among African Americans of reproductive age. Health Educ Behav 2005;32:474-87.

74. Do BN, Tran TV, Phan DT, et al. Health literacy, ehealth literacy, adherence to infection prevention and control procedures, lifestyle changes, and suspected COVID-19 symptoms among health care workers during lockdown: online survey. J Med Internet Res 2020;22:e22894.

75. International Labour Organization (ILO). Working from home: estimating the worldwide potential. Geneva: ILO; 2020.

76. Sen G, Ostlin P, George A. Unequal, unfair, ineffective and inefficient. Gender inequity in health: why it exists and how we can change it. Final report to the WHO Commission on Social Determinants of Health. Geneva: World Health Organization; 2007.

77. Vaezi A, Javanmard SH. Infodemic and risk communication in the era of CoV-19. Adv Biomed Res 2020;9:10. 
78. Diaz de Leon-Martinez L, de la Sierra-de la Vega L, Palacios-Ramirez A, et al. Critical review of social, environmental and health risk factors in the Mexican indigenous population and their capacity to respond to the COVID-19. Sci Total Environ 2020;733:139357.
79. SoleimanvandiAzar N, Irandoost SF, Ahmadi S, et al. Explaining the reasons for not maintaining the health guidelines to prevent COVID-19 in high-risk jobs: a qualitative study in Iran. BMC public health 2021;21:1-15 